\title{
RETRACTED ARTICLE: The role of circuit breakers in the oil futures market
}

\author{
Nicholas Apergis 1
}

Published online: 16 November 2014

(C) Springer Science+Business Media New York 2014

The author has retracted this article due to overlap with previously published articles by Brugler and Linton and Ebrahim et al [1,2]. This retraction supersedes the previous erratum [3]. The author agrees with this retraction.

The online version of this article contains the full text of the retracted article as electronic supplementary material.

Electronic supplementary material The online version of this article (https:/doi.org/10.1007/s12197-0149307-1) contains supplementary material, which is available to authorized users.

Nicholas Apergis

napergis@unipi.gr

1 Department of Economics and Property, Curtin University, Bentley, Australia 